\title{
Metodología de Binford para la Categorización Tafonómica de Marcas de Dientes Animales en Superficies Óseas. Una Revisión con Búsqueda Sistemática
}

\author{
Binford Methodology for Taphonomic Classification of Animal \\ Tooth Marks on Bone Surfaces. A Systematic Search Review
}

Fabiana Scarso-Giaconi ${ }^{1,2}$; Sandra López-Lázaro' ${ }^{1,2}$; Inmaculada Alemán-Aguilera ${ }^{3}$ \& Gabriel M. Fonseca ${ }^{1,2}$

SCARSO-GIACONI, F.; LÓPEZ-LÁZARO, S.; ALEMÁN-AGUILERA, I. \& FONSECA, G. M. Metodología de Binford para la categorización tafonómica de marcas de dientes animales en superficies óseas. Una revisión con búsqueda sistemática. Int. J. Morphol., 38(4):838-844, 2020.

RESUMEN: La Tafonomía, concepto reconocido en paleontología y antropología, permite estimar tiempo y circunstancias de muerte mediante el estudio de condiciones post-mortem y factores relativos a la supervivencia de restos óseos. Entre las diferentes variables tafonómicas, Binford (1981) propuso una categorización de marcas de mordedura, hoy reconocido estándar para estos estudios. Sin embargo, su diseño original en inglés podría llevar a malinterpretaciones o informes erróneos por no poseer una traducción validada. Se realizó una revisión con búsqueda sistemática de artículos en español, sin límite temporal, que citaran a Binford ("Bones: Ancient Men and Modern Myths") en Google Scholar. Fueron incluidos aquellos que mencionaran esta metodología para categorizar marcas de mordedura como agente tafonómico. Se excluyeron tesis, libros, pósters y resúmenes de congresos. Se identificaron 349 documentos y se seleccionaron 83 según criterios. El 65,12 \% refirió sólo a huellas de mordedura animal sin especificar categorías; el 26,51 \% mostró traducciones libres o categorizaciones parciales, y el 8,43 \% realizó categorizaciones sin traducción. La mayor cantidad de citas fueron realizadas por autores de Argentina y España (54,22 \% y 24,10 \% respectivamente, como única filiación). Las categorías con más traducciones libres fueron "pitting" y "puncture" (11 y 8 términos diferentes respectivamente). Si bien la categorización de Binford es citada profusamente en países de habla hispana para definir las marcas de mordedura animal en hueso, la ausencia de transculturización conlleva falta de consenso para la comunidad científica. Más allá de una traducción literal, la adaptación y estandarización de estos términos es necesaria para validar esta metodología.

PALABRAS CLAVE: Tafonomía; Huellas de mordedura; Hueso; Terminología; Transculturización.

\section{INTRODUCCIÓN}

La "Tafonomía” es la ciencia que estudia los procesos sufridos por un fósil desde el momento en que se deposita hasta su recuperación (Olsen, 1980), siendo de gran aporte en disciplinas como la paleontología, arqueología, antropología, zoología y biología. Por tanto, es relevante la información contextual que pueda aportar, y el enfoque multidisciplinario con el que sea realizado su procesamiento. Entre los procesos que definen los cambios tafonómicos sufridos por restos depositados en superficie o enterrados, se encuentran los producidos por animales (intervención por carroñeo). Este proceso tafonómico involucra el uso de la dentición animal para desgarrar, remover, desarticular, roer, masticar, fracturar o consumir tejido blando y/o huesos. El carroñeo puede modificar, dispersar y remover tejido blando o duro, resultando en fragmentación, fracturas y/o huellas de mordedura (Haglund et al., 1988); y generando grandes complicaciones para la patología y antropología forense (Erkol \& Hösükler, 2018).

Una huella de mordedura se define como "un patrón hecho por los dientes (humanos o animales) sobre un sustrato" (Bernstein, 2011). Su morfología depende del tamaño de la especie carroñera, el tamaño de la mandíbula, la fuerza de mordida y sus patrones dentales (Haynes, 1983;

\footnotetext{
${ }^{1}$ Programa de Magíster en Odontología, Facultad de Odontología, Universidad de La Frontera, Temuco, Chile.

${ }^{2}$ Centro de Investigación en Odontología Legal y Forense (CIO), Facultad de Odontología, Universidad de La Frontera, Temuco, Chile.

${ }^{3}$ Departamento de Medicina Legal, Toxicología y Antropología Física, Universidad de Granada, Granada, España.

Trabajo financiado (parcialmente) por la Dirección de Investigación, Universidad de La Frontera, Temuco, Chile.
} 
Young et al., 2015). Las marcas que resultan de una huella de mordedura en tejido óseo, han sido clasificadas y descritas por el arqueólogo estadounidense L. Binford (1981) como "Pits", "Punctures", "Furrows" y "Scores" en el año 1981, en idioma inglés (Tabla I); y su uso en la bibliografía en español es evidente.

En escenarios arqueológicos, el uso de esta categorización ha sido extensamente aplicado para entender la interacción de las especies y su presencia en diferentes contextos. Por otro lado, en escenarios forenses, la aparición de estas marcas en tejido óseo, significa una intervención ante, peri o post mortem entre el individuo de estudio y el carroñero, que deberá ser analizada, entendida e informada (Tokdemir et al., 2014). En muertes post traumáticas, las heridas abiertas serían el punto de inicio de consumo para los carroñeros, y en poco tiempo, podrían complicar la correcta identificación de heridas antemortem (Willey \& Snyder, 1989); como, por ejemplo, encubrir, heridas por armas de fuego, puñaladas por objetos punzantes, o marcas de ligadura (Young et al.).

El uso de la información de la marca de mordedura en conjunto con el conocimiento del comportamiento y patrones específicos de las especies, así como los factores mencionados anteriormente, llevarán a una identificación más específica del taxa y tamaño del carroñero, y si es posible, la especie (Young et al.). Así entonces, los carnívoros generan cambios de estructura en las epífisis de los huesos largos, en procesos vertebrales transversos y espinosos y márgenes distales de costillas, escápula y huesos de la cadera (Haglund et al.).

Ante la necesidad que genera una pericia forense de describir y categorizar los elementos involucrados en una escena que contenga elementos óseos con modificaciones producidas por animales, la terminología de Binford es utilizada y reconocida por los investigadores del área. Sin embargo, la ausencia de transculturización de los términos al español ha llevado a la comunidad científica de habla hispana a la falta de un consenso terminológico cuando deben emitirse reportes de este tipo de análisis. El objetivo de esta revisión es evidenciar el uso de la metodología de Binford para categorizar marcas de mordedura animal en hueso, y revisar su aplicación.

\section{MATERIAL Y MÉTODO}

Se realizó una revisión de la literatura en la base de datos Google Scholar, seleccionando los artículos que citaran el trabajo de Binford "Bones. Ancient men and modern myths" (1981). Éstas se realizaron entre el 4 y 20 de abril de 2018 limitándose a estudios en español donde se citara a Binford para categorizar marcas de mordedura animal como efecto tafonómico. Dentro de las categorías de estudio se consideraron el año de la publicación, la nacionalidad de los autores y la mención de una, todas o ninguna categoría de clasificación. Fueron excluidos los resultados de tesis doctorales, libros, pósters y resúmenes de congresos. Una búsqueda complementaria fue realizada con el objetivo de identificar los documentos en las referencias citadas en esos artículos, siempre enfocando el objetivo del estudio.

\section{RESULTADOS}

Se identificaron 349 artículos como resultado de la búsqueda, de los cuales 82 cumplieron la totalidad de los criterios de inclusión y exclusión y presentaron el contenido requerido. Una búsqueda manual posterior permitió añadir una publicación que reunía similares criterios. En total se obtuvieron 83 artículos para el análisis (Fig. 1).

Fecha de publicación y países filiados por los autores. Se identificaron publicaciones entre los años 1997 y 2017, con un máximo de 10 documentos publicados en cada uno de los años 2010 y 2014. En 2017 se pesquisaron 5 publicaciones. Del total de 83 artículos, el país con más participaciones fue Argentina con 45 (54,22\%) como única filiación, en $4(4,82 \%)$ asociado con Chile (sumando este país además 5 $(6,02 \%)$ publicaciones como única filiación) y $1(1,20 \%)$

Tabla I. Categorías de marcas de mordedura en hueso según Binford (1981).

\begin{tabular}{ll}
\hline \multicolumn{1}{c}{ CATEGORÍA } & DESCRIPCIÓN ORIGINAL \\
\hline Punctures & "Where the bone has collapsed under the tooth, frequently leaving a fairly clear imprint of the \\
tooth" & "Results from gnawing bones rather than eating and pulling meat from the skeleton of the \\
Pitting & "Result from turning the bone against the teeth or dragging the teeth across relatively compact \\
bone. The result is a scarring of the bone commonly linear" & "The effect that repeated jaw action (with either carnassials or canines) produces on relatively \\
cancellous tissue"
\end{tabular}




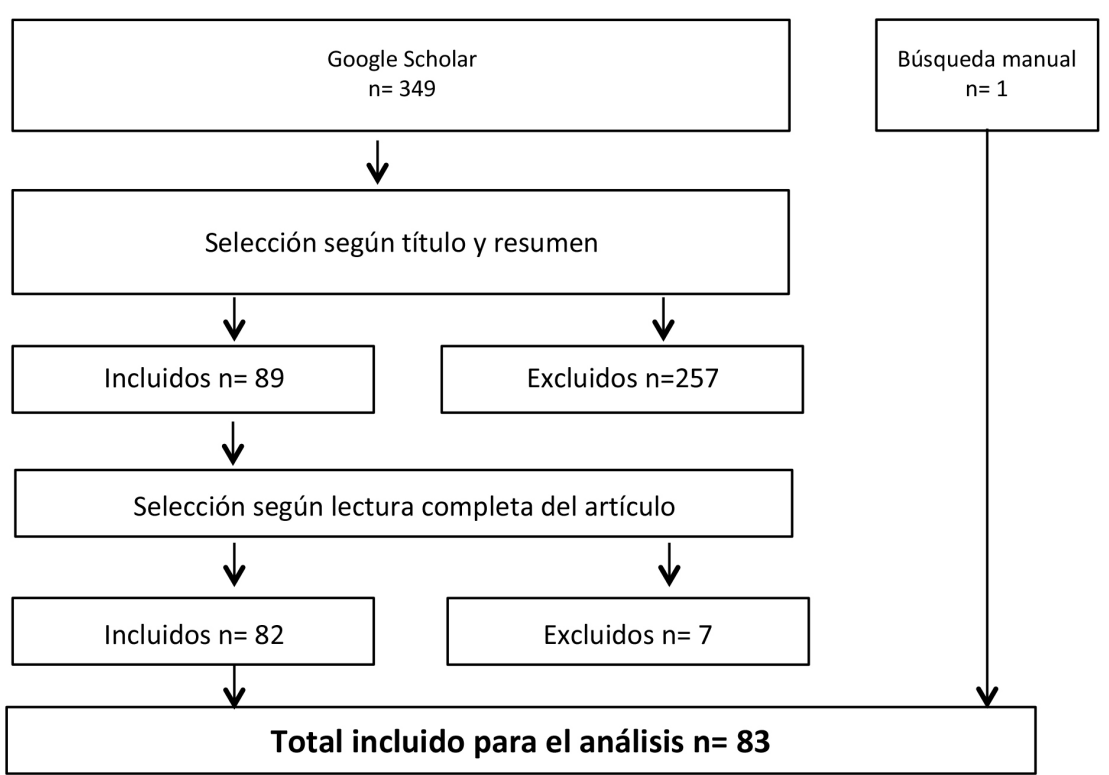

Fig. 1. Flujograma de la revisión de literatura y criterios para selección y exclusión de artículos

asociado con España. El segundo país con más participaciones fue España con $20(24,10 \%)$ como única filiación y $4(4,82 \%)$ en colaboración con Argentina y con otros países europeos (Alemania, Francia y Portugal). Mientras que el máximo de publicaciones de autores argentinos fue de 8 artículos $(9,64)$ en el año 2014, el de autores españoles fue de $7(8,43 \%)$ en el año 2010, lo que da expresión a los dos peaks de esta revisión. Otros países identificados en las filiaciones fueron Francia (1 artículo con filiación única y 2 en colaboraciones), México (1 con filiación única y 1 en colaboración), Alemania, Estados Unidos y Portugal (cada uno con 1 filiación en colaboración) y Cuba y Uruguay (cada uno con 1 filiación única) (Tabla II).

Referencia a categorización de Binford. Del total de los 83 artículos seleccionados, siempre citando la obra de Binford (1981), 54 documentos (65,12\%) sólo refirieron la presencia de marcas de mordedura animal sin realizar categorizaciones, mientras que 7 artículos $(8,43 \%)$ hicieron referencia a las categorías por sus nombres originales en inglés sin traducirlos. Es de destacar que de los 22 artícu$\operatorname{los}(26,51 \%)$ que realizaron traducciones parciales o libres de los rasgos para categorizar las marcas, $3(13,63 \%)$ utilizaron más de un nombre para cada categoría, y sólo $2(9,09$ $\%)$ mencionaron o describieron el rasgo mencionado. Las categorías con mayor variabilidad en sus traducciones fueron "Puncture" y "Pitting", utilizando respectivamente 11 y 8 términos diferentes para hacer mención a ellas.

No se identificó ningún documento que buscara validar traducciones o transculturizaciones de los términos, sobre la base de Binford.

\section{DISCUSIÓN}

Esta revisión evidenció el uso de la categorización de Binford para clasificar las huellas de mordedura animal en tejido óseo. La intención no fue sólo contrastar su uso, sino determinarlo en diferentes contextos, y también expresar la variabilidad de sus traducciones al español. Las diferentes especies de carnívoros poseen variable morfología y fórmula dental, por lo que conocerla es de suma importancia al realizar el análisis forense de este tipo de marcas (De Munnynck \& Van de Voorde, 2002). Las lesiones denominadas "Punctures", son marcas tafonómicas de carácter patognomónico producidas por los dientes caninos de los carnívoros, y pueden ser utilizadas para medir la distancia intercanina, la cual se considera específica para diferentes especies (Rubini et al., 2018). Para los cánidos (Canis lupus familiaris), la variabilidad intra especie es mucho mayor, debido a la gran cantidad de tamaños y razas diferentes (Tedeschi Oliveira et al., 2011), por tanto, es importante contar con esta información. Por otro lado, se ha descrito la similitud de la marca de mordedura animal y humana, y cómo podrían ser mal interpretadas, encubriendo a veces hasta casos de abuso o maltrato infantil (Heit, 2017); ya han sido estudiados los parámetros de diferencias entre una y otra, existiendo similitudes en la distancia inter canina de un humano adulto y un perro (Kayshap et al., 2015), por tanto, no serían concluyentes por sí solas al momento de realizar un informe (Tedeschi Oliveira et al.). Pero, considerando la variabilidad de razas de cánidos, sí se podrían determinar ciertos patrones de mordida por la fuerza de su mandíbula, y patrones de consumo y carroñeo (Murmann et al., 2006; Gidna et al., 2013). Por otro lado, Dominguez-Rodrigo \& Piqueras (2003) proponen una categorización de carnívoros según las medidas de la marca denominada "pit" midiendo el ancho y longitud de la misma; e incluso proponen una categorización de carnívoros según estas medidas. Aparte, Murmann et al. postulan que podría diferenciarse la actividad realizada por carnívoros o felinos si se evalúan en combinación las medidas de las fauces superiores e inferiores.

Probablemente la mayor problemática expuesta por esta revisión es la referida a la gran variabilidad ( $i$ "heterogeneidad", "disenso"?) de la terminología empleada citando a Binford. En el ámbito forense, estas circunstancias ya han sido muy bien expresadas tanto en el área médica 
SCARSO-GIACONI, F.; LÓPEZ-LÁZARO, S.; ALEMÁN-AGUILERA, I. \& FONSECA, G. M. Metodología de Binford para la categorización tafonómica de marcas de dientes animales en superficies óseas. Una revisión con búsqueda sistemática. Int. J. Morphol., 38(4):838-844, 2020.

Tabla II. Resumen de los estudios incluidos en esta revisión.

\begin{tabular}{|c|c|c|}
\hline Autores & País Autores & $\begin{array}{c}\text { Referencia a categorización de } \\
\text { Binford (1981) }\end{array}$ \\
\hline Borrero et al., 1997 & Argentina, Chile & RCTL/P \\
\hline Domínguez-Rodrigo \& de la Torre Sainz, 1999 & España & RNC \\
\hline Monchot, 1999 & Francia & RNC \\
\hline Yravedra Sáinz de los Terreros, 2000 & España & RNC \\
\hline Yravedra Sáinz de los Terreros, 2001 & España & RNC \\
\hline Yravedra Sáinz de los Terreros, 2001 & España & RNC \\
\hline Martin et al., 2004 & Argentina, Chile & RNC \\
\hline Velásquez, 2004 & Chile & RCNT \\
\hline Yravedra Sáinz de los Terreros, 2004 & España & RCNT \\
\hline Savanti et al., 2005 & Argentina, España & RNC \\
\hline Yravedra Sáinz de los Terreros, 2005 & España & RNC \\
\hline Caracotche et al., 2005 & Argentina & RCNT \\
\hline Borrero et al., 2005 & Argentina, Chile & RCTL/P \\
\hline Rindel \& Belardi, 2006 & Argentina & $\mathrm{RNC}$ \\
\hline Mondini, 2006 & Argentina & RCTL/P \\
\hline Cartajena \& Labarca, 2007 & Chile & RNC \\
\hline Chacón Navaro \& Fernández Laso, 2007 & España & RNC \\
\hline López Mendoza, 2007 & Chile & RCTL/P \\
\hline Muñoz, 2008 & Argentina & RNC \\
\hline Izeta, 2008 & Argentina & RNC \\
\hline Cahiza et al., 2008 & Argentina & RNC \\
\hline Yravedra Sáinz de los Terreros, 2008 & España & RNC \\
\hline Labarca et al., 2008 & Chile & RCNT \\
\hline Muñoz et al., 2008 & Argentina & RCTL/P \\
\hline Labarca, 2009 & Argentina & RNC \\
\hline Llago, 2009 & Argentina & RNC \\
\hline Martin et al., 2009 & España & RNC \\
\hline Cordero, 2009 & Argentina & RCTL/P \\
\hline Fernández \& Forlano, 2009 & Argentina & RCTL/P \\
\hline Scheifler, 2010 & Argentina & RNC \\
\hline Lanza, 2010 & Argentina & RNC \\
\hline Martín \& San Román, 2010 & Chile & $\mathrm{RNC}$ \\
\hline Yravedra Sáinz de los Terreros et al., 2010 & España & RNC \\
\hline Yravedra Sáinz de los Terreros, 2010 & España & RNC \\
\hline Yravedra Sáinz de los Terreros \& Gómez Castanedo, 2010 & España & $\mathrm{RNC}$ \\
\hline Yravedra Sáinz de los Terreros \& Gómez Castanedo, 2010 & España & $\mathrm{RNC}$ \\
\hline Huget et al., 2010 & España & RCTL/P \\
\hline López et al.. 2010 & España & $\mathrm{RCTL} / \mathrm{P}$ \\
\hline Canals et al., 2010 & Francia, España & RCTL/P \\
\hline Flensborg et al., 2011 & Argentina & RNC \\
\hline Cruz et al., 2011 & Argentina & RNC \\
\hline Massigoge, 2011 & Argentina & RNC \\
\hline Medina et al.. 2011 & Argentina & RCTL/P \\
\hline Dantas, 2011 & Argentina & RCTL/P \\
\hline Serra \& Benito, 2011 & España & RCTL/P \\
\hline Cornaglia Fernández et al.. 2012 & Argentina & RNC \\
\hline Ortiz \& Urquiza, 2012 & Argentina & RNC \\
\hline Hernández, 2012 & Argentina & RNC \\
\hline
\end{tabular}




\begin{tabular}{|c|c|c|}
\hline Autores & País Autores & $\begin{array}{c}\text { Referencia a categorización de } \\
\text { Binford (1981) }\end{array}$ \\
\hline Fuentes-Mucherl et al., 2012 & Argentina, Chile & $\mathrm{RNC}$ \\
\hline Margalef, 2012 & España & $\mathrm{RNC}$ \\
\hline García \& del Papa, 2012 & Argentina & RCNT \\
\hline Doval \& Tapia, 2012 & Argentina & RCTL/P \\
\hline Day Pilaría et al., 2013 & Argentina & $\mathrm{RNC}$ \\
\hline Lanza, 2013 & Argentina & $\mathrm{RNC}$ \\
\hline Gonzalez, 2013 & Argentina & $\mathrm{RNC}$ \\
\hline Yravedra Sáinz de los Terreros et al., 2013 & España & RCNT \\
\hline Gusi et al., 2013 & España, Alemania & RCTL/P \\
\hline Martínez et al., 2014 & Argentina & $\mathrm{RNC}$ \\
\hline Barboza \& Martini, 2014 & Argentina & $\mathrm{RNC}$ \\
\hline Barboza, 2014 & Argentina & $\mathrm{RNC}$ \\
\hline Nasif \& Miguez, 2014 & Argentina & $\mathrm{RNC}$ \\
\hline Day Pilaría et al., 2014 & Argentina & $\mathrm{RNC}$ \\
\hline Pérez Iglesias \& Valcárcel Rojas, 2014 & Cuba & $\mathrm{RNC}$ \\
\hline Machause López et al., 2014 & España & $\mathrm{RNC}$ \\
\hline Rafuse et al., 2014 & Argentina & RCTLP \\
\hline Massigoge et al., 2014 & Argentina & RCTL/P \\
\hline Urquiza \& Aschero, 2014 & Argentina & RCTLP \\
\hline Cruz et al., 2015 & Argentina & $\mathrm{RNC}$ \\
\hline Vásquez, 2015 & Argentina & $\mathrm{RNC}$ \\
\hline Muñoz, 2015 & Argentina & $\mathrm{RNC}$ \\
\hline Hernández, 2015 & Argentina & $\mathrm{RNC}$ \\
\hline García Añiño, 2015 & Argentina & $\mathrm{RNC}$ \\
\hline Fernández-Laso et al., 2015 & España, Francia, & $\mathrm{RNC}$ \\
\hline Cárdenas Cervantes \& Götz, 2015 & Mexico & $\mathrm{RNC}$ \\
\hline Dantas \& Srur, 2015 & Argentina & RCTL/P \\
\hline Barboza, 2016 & Argentina & $\mathrm{RNC}$ \\
\hline Mercolli \& Taboada, 2016 & Argentina & $\mathrm{RNC}$ \\
\hline Navarro, 2016 & Argentina & RCNT \\
\hline Pérez Roldán et al., 2017 & Mexico, Estados & $\mathrm{RNC}$ \\
\hline Moreno, 2017 & Uruguay & $\mathrm{RNC}$ \\
\hline Banfi, 2017 & Argentina & RCTL/P \\
\hline Tavarone et al., 2017 & Argentina & RCTL/P \\
\hline González-Rábana et al., 2017 & España & RCTL/P \\
\hline
\end{tabular}

$(\mathrm{RNC}=$ Rasgos no categorizados; RCNT $=$ Rasgos categorizados no traducidos; RCTL/P= Rasgos categorizados con traducciones libres o parciales)

(Fogarasi, 2011) como odontológica (Fonseca et al., 2011), siempre coincidiendo en lo dificultoso de suscribir informes periciales con interpretaciones absolutamente dependientes de los términos empleados y potenciales pérdidas de información precisa para la elaboración de dictámenes. Sorg \& Haglund (2002), afirman que el uso y valor de algunos términos difiere entre disciplinas: si bien una palabra puede ser bien entendida y funcionalmente adecuada, una vez aplicada a un contexto disciplinario diferente, su empleo puede llevar a malentendidos y errores de suposición. Aunque en el ámbito médico legal de habla hispana para la descripción de lesiones, existe cierto consenso "no escrito" en citar la propuesta terminológica del catedrático español Juan Antonio Gisbert Calabuig (Villanueva Cañadas, 2019), la expresión de injurias a nivel óseo -incluidas las tafonómicas-, aun permanece en esta heterogeneidad. Nuevamente Sorg \& Haglund expresan que en el ámbito de la tafonomía forense, el traslado de términos provenientes de la anatomía hacia la medicina y luego a la antropología forense afecta sus inferencias y significación forense para determinados contextos. Esta revisión expone además que en países de habla hispana como Argentina, Chile y España, 
la categorización de Binford es gold standard para describir las lesiones encontradas en tejido óseo, y es utilizada por los servicios médicos de salud encargados de realizar los informes periciales. La variabilidad de sinónimos para cada categoría que evidencia esta revisión, deja en claro la falta de consenso frente a la materia, y las inminentes necesidades de hablar un mismo lenguaje y de comprender el significado de lo que se está informando a un nivel morfológico $\mathrm{y}$, evidentemente, pericial. Se ha mencionado que, siendo muy frecuente la utilización de instrumentos diseñados en otro idioma y no validados (lo que limita considerablemente la interpretación de datos), es importante el desarrollo de nuevos instrumentos o modificarlos lo suficiente como para validarlos al lenguaje nativo de quien lo empleará, proceso denominado "de adaptación transcultural" (Lauffer et al., 2013). Consideramos que la implementación de un protocolo de adaptación transcultural al español de la categorización de Binford que demuestre sus propiedades de medición para ser evaluado y reevaluado en diferentes situaciones y por diferentes investigadores, permitirá resolver un dilema terminológico actualmente carente de estándares, aunándose a los objetivos de resolver el cómo definir y describir este tipo de lesiones óseas, si bien específicas, de gran valor a la investigación en tafonomía zoológica, antropológica y forense.

\section{CONCLUSIONES}

Las marcas de mordedura animal en tejido óseo son una fuente de información muy valiosa si se sabe clasificar e interpretar. Por tanto, deben ser investigadas en conjunto por odontólogos, médicos, médicos veterinarios, antropólogos y biólogos para llegar a un correcto diagnóstico de ellas. Para esto, es necesario que la clasificación de estas marcas sea interpretada de la misma manera entre los profesionales de habla hispana. Una correcta traducción y transculturización de esta clasificación, unificará la forma en que se categorizan las marcas, y llevará a la realización de informes periciales que se comprendan de la misma forma a lo largo del mundo.

SCARSO-GIACONI, F.; LÓPEZ-LÁZARO, S.; ALEMÁNAGUILERA, I. \& FONSECA, G. M. Binford methodology for taphonomic classification of animal tooth marks on bone surfaces. A systematic search review. Int. J. Morphol., 38(4):838-844, 2020.

SUMMARY: Taphonomy, a concept recognized in paleontology and anthropology, allows the estimation of time and circumstances of death by studying post-mortem conditions and factors related to the survival of bone remains. Among the different taphonomic variables, Binford (1981) proposed a categorization of bite marks, now recognized as standard for these studies. However, its original design in English could lead to misinterpretations or erroneous reports for not having a validated translation. A scoping review was conducted for articles in Spanish, without a time limit, that cited Binford ("Bones: Ancient Men and Modern Myths") in Google Scholar. Those who mentioned this methodology to categorize bite marks as taphonomic agent were included. Theses, books, posters and conference proceedings were excluded. We identified 349 documents, and 83 were selected according to criteria. $65.12 \%$ referred only to animal bite marks without specifying categories; $26.51 \%$ showed free translations or partial categorizations, and $8.43 \%$ made categorizations without translation. The largest number of citations were made by authors from Argentina and Spain (54.22\% and $24.10 \%$ respectively, as the only affiliation). The categories with the most free translations were "pitting" and "puncture" (11 and 8 different terms respectively). While Binford's categorization is widely cited in Spanish-speaking countries to define animal bite marks in bone, the absence of transculturation implies a lack of consensus for the scientific community. Beyond a literal translation, the adaptation and standardization of these terms is necessary to validate this methodology.

KEY WORDS: Taphonomy; Bite marks; Bone; Terminology; Transculturation.

\section{REFERENCIAS BIBLIOGRÁFICAS}

Bernstein, M. The Nature of Bitemarks. En: Dorion, R. (Ed.). Bitemark Evidence: A Color Atlas and Text. $2^{\mathrm{a}}$ ed. Boca Raton, CRC Press, 2011. pp.53-65.

Binford, L. R. Bones: Ancient Men and Modern Myths. Nueva York, Academic Press, 1981. pp.46-9.

De Munnynck, K. \& Van de Voorde, W. Forensic approach of fatal dog attacks: a case report and literature review. Int. J. Legal Med., 116(5):295-300, 2002.

Dominguez-Rodrigo, M. \& Piqueras, A. The use of tooth pits to identify carnivore taxa in tooth-marked archaeofaunas and their relevance to reconstruct hominid carcass processing behaviours. J. Archaeol. Sci., 30(11):1385-91, 2003.

Erkol, Z. \& Hösükler, E. Postmortem Animal Attacks on Human Corpses. En: Dogan, K. H. (Ed.). Post Mortem Examination and Autopsy Current Issues from Death to Laboratory Analysis. Rijeka, IntechOpen, 2018.

Fogarasi, K. Terminological problems and information missing in descriptions of injuries in the Hungarian forensic medical discourse. A. M. M., 57(3):183-185, 2011.

Fonseca, G. M.; Salgado Alarcón, G. \& Cantín, M. Lenguaje odontológico forense e identificación: obstáculos por falta de estándares. Rev. Esp. Med. Legal, 37(4):162-8, 2011.

Gidna, A.; Yravedra, J. \& Dominguez-Rodrigo, M. A cautionary note on the use of captive carnivores to model wild predator behavior: a comparison of bone modification patterns on long bones by captive and wild lions. J. Archaeol. Sci., 40(4):1903-10, 2013.

Haglund, W. D.; Reay, D. T. \& Swindler, D. R. Tooth mark artifacts and survival of bones in animal scavenged human skeletons. J. Forensic Sci., 33(4):985-97, 1988.

Haynes, G. Frequencies of spiral and green-bone fractures on ungulate limb bones in modern surface assemblages. Am. Antiq., 48(1):10214,1983 
SCARSO-GIACONI, F.; LÓPEZ-LÁZARO, S.; ALEMÁN-AGUILERA, I. \& FONSECA, G. M. Metodología de Binford para la categorización tafonómica de marcas de dientes animales en superficies óseas. Una revisión con búsqueda sistemática. Int. J. Morphol., 38(4):838-844, 2020.

Heit, O. F. J. Mordedura de perro imitando una lesión por abuso infantil. Rev. Bras. Odontol. Leg., 4(2):104-109, 2017.

Kayshap, B.; Anand, S.; Reddy, S.; Sahukar, S. B.; Supriya, N. \& Pasupuleti, S. Comparison of the bite mark pattern and intercanine distance between humans and dogs. J. Forensic Dent. Sci., 7(3):175-9, 2015.

Lauffer, A.; Solé, L.; Bernstein, S.; Lopes, M. H. \& Francisconi, C. F. Cómo minimizar errores al realizar la adaptación transcultural y la validación de los cuestionarios sobre calidad de vida: aspectos prácticos. Rev. Gastroenterol. Mex., 78(3):159-76, 2013.

Murmann, D. C.; Brumit, P. C.; Schrader, B. A. \& Senn, D. R. A comparison of animal jaws and bite mark patterns. J. Forensic Sci., 51(4):846-60, 2006.

Olsen, E. C. Taphonomy: Its History and Role in Community Evolution. En: Behrensmeyer, A. K. \& Hill, A. P. (Eds.). Fossils in the Making: Vertebrate Taphonomy and Paleoecology. Chicago, The University of Chicago Press, 1980. pp.5-19.

Rubini, S.; Barbieri, S.; Gaudio, R. M.; Govoni, G.; Berna, G. R.; Fico, R.; Lorenzini, R.; Fontana, M. C.; Taddei, R.; Tassinari, M.; et al. Veterinary forensic sciences to solve a fatal case of predation on flamingos (Phoenicopterus roseus). Vet. Ital., 54(2):175-80, 2018.

Sorg, M. H. \& Haglund, W. D. Advancing Forensic Taphonomy: Purpose, Theory, and Process. En: Haglund, W. D. \& Sorg, M. H. (Eds.). Advances in Forensic Taphonomy - Method, Theory, and Archaeological Perspectives. Boca Raton, CRC Press, 2002.

Tedeschi Oliveira, S. V.; Trigueiro, M.; Oliveira, R. N. \& Melani, R. F. Intercanine distance in the analysis of bite marks: a comparison of human and domestic dog dental arches. J. Forensic Odontostomatol., 29(1):30-6, 2011.

Tokdemir, M. B.; Çetin, G.; Sam, B.; Özer, E.; Yıldırım, A. \& Bütüns, C. The challenges in autopsy cases exposed to animal attack. Med. Sci., 3(2):1109-23, 2014.

Villanueva Cañadas, E. Gisbert Calabuig. Medicina Legal y Toxicología. $7^{\mathrm{a}}$ ed. Barcelona, Elsevier, 2019.

Willey, P. \& Snyder, L. M. Canid modification of human remains: implications for time-since-death estimations. J. Forensic Sci., 34(4):894-901, 1989

Young, A.; Stillman, R.; Smith, M. J. \& Korstjens, A. H. Scavenger speciestypical alteration to bone: using bite mark dimensions to identify scavengers. J. Forensic Sci., 60(6):1426-35, 2015.
Dirección para correspondencia:

Dr. Gabriel M. Fonseca

Centro de Investigación en Odontología Legal y Forense

Facultad de Odontología

Universidad de La Frontera

Francisco Salazar 01145

Temuco

CHILE

Email: gabriel.fonseca@ufrontera.cl

Recibido : 09-12-2019

Aceptado: 11-02-2020 